\title{
Infection of Drosophila suzukii with the obligate insect-pathogenic fungus Entomophthora muscae
}

\author{
Paul G. Becher ${ }^{1}$ (I) - Rasmus E. Jensen ${ }^{2,3} \cdot$ Myrsini E. Natsopoulou $^{2} \cdot$ \\ Vasiliki Verschut $^{1} \cdot$ Henrik H. De Fine Licht $^{2}$ (D)
}

Received: 30 May 2017/Revised: 21 August 2017/ Accepted: 31 August 2017/Published online: 9 September 2017

(C) The Author(s) 2017. This article is an open access publication

\begin{abstract}
Physiological constraints restrict specialist pathogens from infecting new hosts. From an applied perspective, a narrow host range makes specialist pathogens interesting for targeting specific pest insects since they have minimal direct effects on non-target species. Entomopathogenic fungi of the genus Entomophthora are dipteran-specific but have not been investigated for their ability to infect the spotted wing drosophila (SWD; Drosophila suzukii) a fruit-damaging pest invasive to Europe and America. Our main goal was to study whether SWD is in the physiological host range of the entomophthoralean species E. muscae. We investigated pathogenicity and virulence of $E$. muscae towards its main natural host, the housefly Musca domestica, and towards SWD. We found that E. muscae readily infected and significantly reduced survival of SWD by $27.3 \%$ with the majority of flies dying 4-8 days post-exposure. In comparison with SWD, infection of the natural host $M$. domestica resulted in an even higher mortality of $62.9 \%$ and larger conidial spores of $E$.
\end{abstract}

Communicated by A. Biondi.

Paul G. Becher

paul.becher@slu.se

$\triangle$ Henrik H. De Fine Licht

hhdefinelicht@plen.ku.dk

1 Chemical Ecology Unit, Department of Plant Protection Biology, Swedish University of Agricultural Sciences, Box 102, 23053 Alnarp, Sweden

2 Section for Organismal Biology, Department of Plant and Environmental Sciences, University of Copenhagen, Thorvaldsensvej 40, 1871 Frederiksberg, Denmark

3 Section for Entomology and Plant Pathology, Department of Agroecology, Aarhus University, Forsøgsvej 1, 4200 Slagelse, Denmark muscae, reflecting the physiological constraints of the pathogen in the atypical host. We demonstrated that pathogens of the E. muscae species complex that typically have a narrow natural host range of one or few dipteran species are able to infect SWD, and we described a new method for in vivo transmission and infection of an entomophthoralean fungus to SWD.

Keywords Entomopathogen · Fly · Fungal pathogen · Insect pest $\cdot$ Spotted wing drosophila

\section{Key message}

- Dipteran-specific pathogens are potential agents to control the fruit-damaging Drosophila suzukii.

- This is the first report of the obligate insect-pathogenic fungus Entomophthora muscae being able to infect and kill male and female D. suzukii.

- An even higher virulence towards the natural host Musca domestica and differences in spore morphology may reflect physiological constraints of the tested $E$. muscae isolate when infecting D. suzukii.

- Entomophthora species like the dipteran-specific E. muscae have a potential for biological control of $D$. suzukii.

\section{Introduction}

The Asian spotted wing drosophila (SWD; Drosophila suzukii) is an invasive and serious economic pest in fruit and berry. Since growers became aware of a SWD invasion in Southern Europe and the Eastern USA in 2008, the fly 
expanded its geographic range dramatically within Europe and the Americas (Asplen et al. 2015).

During geographic expansion, invasive insects come into contact with previously un-encountered pathogens that try to exploit the new species as a host. However, virulent pathogens, parasites and predators can be sparse or unable to regulate populations of pest species at early states of invasion as exemplified by the devastating dispersal of SWD. Pathogens that are able to develop diseases in a new invasive pest consequently are of potential value to suppress population densities and thus the impact of the pest. Active suppression of pest populations can be approached through different strategies of biological control ranging from measures that protect or enhance specific antagonists in the environment, to the intentional release of control agents (Eilenberg et al. 2001). Antagonists such as entomopathogenic fungi are generally accepted as a safer alternative to chemical insecticides, although direct and indirect ecological effects on non-target organisms are common and need to be considered in risk assessment of any control measure (Flexner et al. 1986; Cory and Myers 2000; Goettel and Hajek 2000; Shah and Pell 2003). Negative effects on beneficial and other non-target arthropods were for example shown for entomopathogenic fungi from the genera Metarhizium and Beauveria (Vestergaard et al. 2003).

Entomopathogenic fungi are common in nature, have significant impact on insect populations and are successfully applied as biological control agents (Hajek and St. Leger 1994; Goettel et al. 2005; Wang and Wang 2017). Insect-pathogenic fungi differ in the natural range of host species they infect and are often designated as generalists or specialists (Boomsma et al. 2014). The wide range of host species used by generalist insect-pathogenic fungi, such as many species within the genera Metarhizium and Beauveria, imply that these fungi are likely to make 'hostshifts' onto newly encountered hosts. Consequently, hypocrealean fungi such as $M$. robertsii and B. bassiana are commonly applied for insect control (Ferron 1981; Meyling and Eilenberg 2007), and commercially available fungal biological control agents based on generalist entomopathogenic fungi have been studied for control of SWD (Woltz et al. 2015; Cossentine et al. 2016; Cuthbertson and Audsley 2016). Collectively these studies show that SWD can be infected and killed by several different insectpathogenic fungi. In particular, a recent study showed high insecticidal activity of Metarhizium brunneum when applied in specifically designed lures to infect and kill SWD (Yousef et al. 2017).

Because of limitations in time-to-kill and difficulty with application of infective conidia, insect-pathogenic fungi are generally used in combination with other measures as part of an integrated pest management (IPM) strategy
(Haye et al. 2016; Shah and Pell 2003). To avoid disruption of current IPM strategies in fruit and berry control that for example may involve non-target effects on predators and parasitoids, pest-specific pathogens would be desirable but have so far not been explored for biological control of SWD (Cuthbertson and Audsley 2016; Hamby and Becher 2016; Yousef et al. 2017). Diptera-infecting entomophthoralean fungi (Jensen et al. 2006; Vega et al. 2012) are known to cause natural epizootics, killing large numbers of insects and can decimate pest populations (Roberts and Humber 1981). Entomophthoralean fungi in the Entomophthora muscae species complex are morphologically distinguishable based on the number of nuclei in conidia and include E. muscae, E. schizophorae and E. syrphi (Keller et al. 1999; Jensen et al. 2006, 2009). Each species is, in contrast to generalist hypocrealean fungi such as $M$. robertsii and B. bassiana, considered to have narrow natural host ranges. Within Entomophthora species, individual populations are genetically distinct as for example isolates of E. muscae from cabbage fly (Delia radicum) are genetically distinct from E. muscae isolates from house flies (Musca domestica) (De Fine Licht et al. 2017; Jensen et al. 2001). Despite high specificity, isolates of several Entomophthora species are capable of infecting other species of diptera than the natural host (Jensen et al. 2006). Entomophthora schizophorae (isolate originally described as E. muscae) from housefly (M. domestica) is for example able to infect the common fruit fly $(D$. melanogaster $)$ at low prevalence (Steinkraus and Kramer 1987; Keller 2007).

Here, our main goal was to investigate whether SWD is in the physiological host range of the entomophthoralean fungus, Entomophthora muscae s. str. (here after called $E$. muscae), which is an important natural enemy of the common housefly, Musca domestica (Kalsbeek et al. 2001). E. muscae is an obligate insect-pathogen that grows as protoplasts inside the fly host. After typically ca. 6-7 days, E. muscae takes over the behaviour of infected hosts and forces them to seek out elevated positions. The host is eventually killed in a characteristic posture with wings spread away from the abdomen, while E. muscae grows out through the intersegmental membranes in the abdomen where it releases infective conidia (Gryganskyi et al. 2017; Hansen and De Fine Licht 2017). Entomophthora muscae causes natural epizootics in housefly populations (Kalsbeek et al. 2001), and here we explored the infectivity of E. muscae towards SWD. In the laboratory, we tested for infection of SWD with E. muscae by direct exposure to sporulating housefly cadavers and documented pathogenicity, virulence and conidia morphology of $E$. muscae-infected SWD. 


\section{Materials and methods}

\section{Isolates and flies}

House flies (M. domestica, strain: 772a) were provided as pupae from the Department of Agroecology, Aarhus University, Denmark. Flies of SWD (D. suzukii) originated from a laboratory strain maintained at SLU, Alnarp on a cornmeal diet (Revadi et al. 2015). Entomophthora muscae isolate hhdfl130914-01, that was originally obtained from a dead infected $M$. domestica collected in a cow byre near Slangerup, Sealand, Denmark (Hansen and De Fine Licht 2017), and is deposited in the insect-pathogenic fungal culture collection at Department of Plant and Environmental Sciences, University of Copenhagen (acc. no. KVL14-115). The E. muscae isolate was maintained in vivo by continuous infections in house flies as previously described (De Fine Licht et al. 2017). Briefly, house flies were kept in groups of 20-40 flies of mixed sex in containers with diameter: $7.5 \mathrm{~cm}$, height: $8 \mathrm{~cm}$. Containers were closed with insect net and administered with water and dry yeast and sugar mixed 1:6 and kept at $21 \pm 1{ }^{\circ} \mathrm{C}$. For infection, three fresh (dead $<12$ h) E. muscae-sporulating fly cadavers actively discharging conidia were placed at the top of the container for $24 \mathrm{~h}$ at ca. $100 \%$ humidity. After 7 days' post-exposure, dead, infected and sporulating fly cadavers were removed from containers and used to infect new healthy flies.

\section{Experimental set-up}

Adult 3-day-old SWD flies were exposed to fresh $M$. domestica cadavers infected with E. muscae. For infecting SWD, two dead sporulating housefly cadavers were fixed with Vaseline underneath the cotton lid inside a Drosophila food vial for $24 \mathrm{~h}$. Control treatments consisted of the exact same set-up, except two uninfected housefly cadavers were fixed at the lid with Vaseline. Each Drosophila food vial contained 12-27 unmated male or female SWD flies, with eight replicate vials per treatment. Two strips of filter paper were added within each vial to facilitate climbing of infected flies. Vials were kept at room temperature $\left(23{ }^{\circ} \mathrm{C} \pm 2\right.$ ), with a photoperiod of $12: 12$ (L:D). Number of dead SWD flies and inspection of cadavers for presence of external fungal growth and general observations were recorded daily for 10 days. To obtain comparable infectivity measurements for E. muscae infections in house flies, vials of diameter: $7.5 \mathrm{~cm}$, height: $8 \mathrm{~cm}$ with $28-46$ house flies were similarly exposed for $24 \mathrm{~h}$ to two sporulating $E$. muscae-infected housefly cadavers. Number of dead house flies and inspection of cadavers for presence of external fungal growth were recorded daily for 10 days as described for SWD.

\section{Conidia exposure dosage and conidia morphology}

The exposure dosage of E. muscae conidia during the 24-h infection scheme was calculated based on eleven sporulating housefly cadavers placed individually over a $1-\mathrm{ml}$ solution containing $1 \%$ Triton-X and $0.2 \%$ maleic acid to prevent germination of discharged conidia (Hajek et al. 2012). Following 24-h exposure, conidia were counted using a hemocytometer placed under a microscope. To examine conidia morphology in the two different hosts, $E$. muscae-infected cadavers of house flies and SWD were placed on microscope slides at high humidity to induce discharge of conidia onto the microscope slides. Microscope slides with conidia were stained with aceto-orcein and examined with a microscope at $100 \times$ magnification. Length, width and number of nuclei within individual conidia from housefly and SWD cadavers were measured.

\section{Statistical analyses}

Generalized linear models (GLMs) with a binomial distribution were used to analyse the effect of the fungus on the proportion of overall mortality of adult flies. The models included treatment (E. muscae application vs. control), species (D. suzukii vs. M. domestica) and sex of the flies as explanatory variables. Model selection was performed using likelihood ratio tests based on X2 and Akaike's information criterion in a stepwise backward selection process from full models testing main effects and two-way interactions between the explanatory variables. Pairwise comparisons were performed using Tukey's HSD post hoc test, with a Bonferroni correction.

In order to test the effect of E. muscae on the survival of D. suzukii and M. domestica in a time dependent manner the Cox proportional hazards $(\mathrm{PH})$ regression model (Cox 1972) was used. Due to very low percentage of mortality in the control groups of both fly species, which resulted in high levels of censored data, these groups were excluded and survival was analysed as a function of species and sex of the flies. Differences in number of nuclei between $E$. muscae conidia from house flies and fruit flies were analysed with a Wilcoxon rank-sum test, whereas conidia differences in length, width and aspect ratio were analysed using Student's $t$ tests after log-transforming data. All analyses were carried out in $\mathrm{R}$ (v. 3.3.0; $\mathrm{R}$ Core Team 2013) using the packages car, multcomp and survival. 


\section{Results}

\section{Entomophthora muscae infections of $D$. suzukii}

A significant effect of treatment $\left(\chi^{2}=130.027, \mathrm{df}=1\right.$, $P<0.001)$ and species $\left(\chi^{2}=37.696\right.$, df $\left.=1, P<0.001\right)$ was observed on the overall mortality of flies, while sex did not affect the response variable $\left(\chi^{2}=3.316\right.$, $\mathrm{df}=1$, $P=0.068)$. All pairwise comparisons revealed significant differences $(P<0.001)$ except from the comparison between the control groups of the two species $(z=0.263$, $P=0.993$ ) (Fig. 1). From a total of 205 SWD flies exposed to infected $M$. domestica cadavers, 56 died within 10 days, from which, $53.6 \%$ (C.I. 39.7-67.0\%) developed visible external mycelium (Fig. 2). Moreover, in several SWD infection induced characteristic behavioural symptoms with flies climbing elevated positions where they died with wings raised above the body confirming the involvement of the fungus. However, the timing of this behavioural manipulation and death was less synchronized in SWD than house flies, with SWD starting to die earlier than M. domestica but with mortality distributed over more days (Fig. 1). In the respective group of $M$. domestica, from a total of 124 flies exposed to sporulating $M$. domestica cadavers, 78 died, all of which developed visible conidia. Analysis with the Cox PH regression model revealed a significant effect of species on the survival of flies exposed to infected $M$. domestica cadavers $\left(\chi^{2}=32.5794, \mathrm{df}=1\right.$, $P<0.001$ ), while sex of the flies was marginally nonsignificant $\left(\chi^{2}=3.5836, \mathrm{df}=1, P=0.0584\right)$.

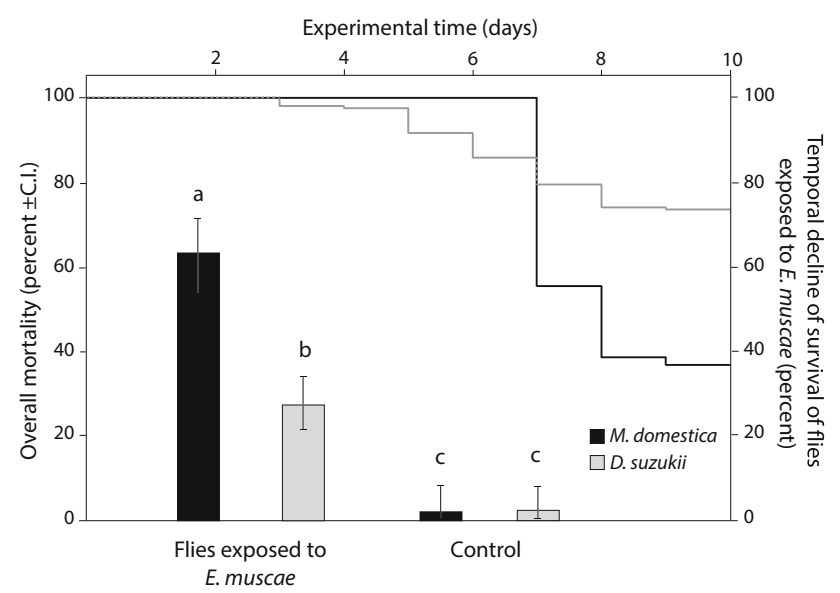

Fig. 1 Percentage of overall mortality over 10 days (bars \pm SE) and temporal decline of survival (lines) in M. domestica (black) and $D$. suzukii (grey) following $24 \mathrm{~h}$ exposure to housefly cadavers with $E$. muscae conidiospores and uninfected control cadavers. Exposure to E. muscae conidia had a significant effect on both $M$. domestica and D. suzukii survival. The letters above each bar denote significantly different overall mortality percentage

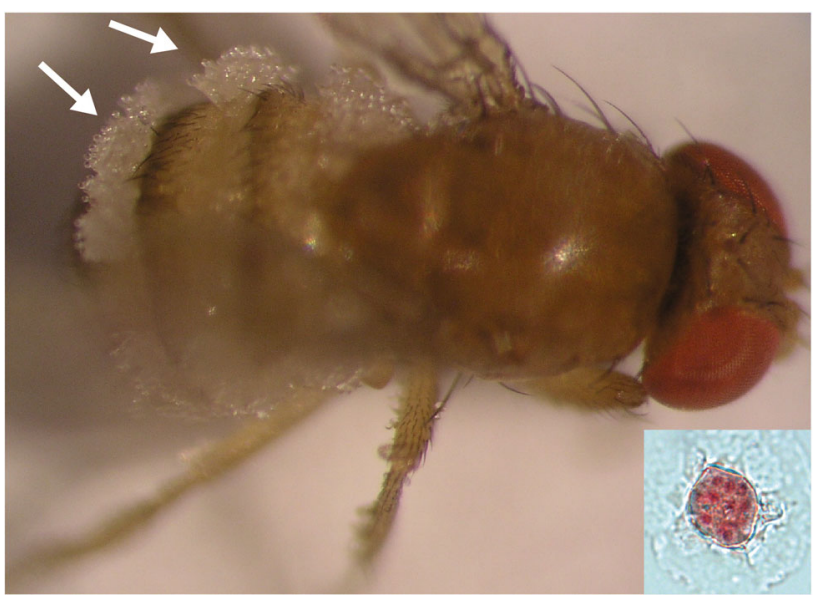

Fig. 2 Abdomen of dead D. suzukii with conidiophores of E. muscae growing out between the tergites and sclerites of the abdomen. Insert shows an E. muscae spore from D. suzukii with the characteristic Entomophthoralean oval shape of a rounded base with a pointed apex. Several nuclei can be seen inside

\section{Conidia exposure dosage and morphology}

In our experimental set-up, a single $E$. muscae-sporulating housefly cadaver produces $2.25 \times 10^{6} \pm 3.46 \times 10^{5}$ conidia (mean $\pm \mathrm{SE}, N=11$ ) during the first $24 \mathrm{~h}$. The SWD and $M$. domestica vials and containers were therefore exposed to a minimum dosage of $4.50 \times 10^{6}$ conidia over the $24 \mathrm{~h}$. There was no difference in number of nuclei between conidia from $E$. muscae when infecting the natural host $M$. domestica and the experimental host SWD ( $W=149.5$, $p=0.249$, Table 1). In contrast, mean length and width of the conidia were significantly smaller on SWD than on $M$. domestica $(t=2.66, \mathrm{df}=37.4, \quad p=0.012 ; t=5.08$, $\mathrm{df}=38.0, p<0.001$, respectively). The shape of conidia measured as the aspect ratio between length and width of the conidia was similarly significantly different between conidia from $E$. muscae when infecting the natural host $M$. domestica and the experimental host SWD $(t=4.37$, $\mathrm{df}=27.9, p<0.001)$, with a wider range of aspect-ratios present in conidia from SWD (Table 1).

\section{Discussion}

The spotted wing drosophila is a most prominent example of insect species that currently invade new geographic regions where they become pests through fast increase in distribution and abundance. Management of invasive pests is a challenge that requires understanding of physiological and ecological mechanisms underlying their dispersal and invasion, and the development of tools to control their impact in natural and agricultural systems (Cini et al. 2014; Hamby et al. 2016). 
Table 1 Spore morphology of E. muscae on the natural host (M. domestica) and the experimental host (D. suzukii)

\begin{tabular}{lllll}
\hline Host species & Mean no. of nuclei & Mean length $(\mu \mathrm{m})$ & Mean width $(\mu \mathrm{m})$ & Mean aspect ratio \\
\hline$M$. domestica & $12.6 \pm 0.3(11-16)$ & $30.4 \pm 0.5(26.2-34.5)$ & $25.9 \pm 0.4(23.2-29.6)$ & $1.17 \pm 0.01(1.11-1.23)$ \\
$D$. suzukii & $13.3 \pm 0.5^{\mathrm{a}}(11-16)$ & $28.5 \pm 0.6(25.2-36.8)$ & $22.8 \pm 0.4(19.7-27.6)$ & $1.25 \pm 0.02(1.15-1.39)$ \\
$t$ test $(t)$ /Wilcoxon & $W=149.5$ & $t=2.66, \mathrm{df}=37.4$ & $t=5.08, \mathrm{df}=38.0$ & $t=4.37, \mathrm{df}=27.9^{\mathrm{b}}$ \\
$(W)$ & $p=0.249$ & $p=0.012$ & $p<0.001$ & $p<0.001$ \\
\hline
\end{tabular}

The mean $(n=20)$ with standard error of the mean and the range in brackets are given

${ }^{\text {a }}$ Nuclei could only be counted in 12 E. muscae spores from D. suzukii

${ }^{\mathrm{b}}$ Aspect ratios were log-transformed to normalize data before performing Student's $t$ test

The release from natural enemies like pathogens and predators is regarded as an important factor contributing to the establishment of invasive species in new habitats (Keane 2002; Comont et al. 2014). Pathogens, as one category of natural antagonists, consequently are applied to counteract enemy release. Entomopathogenic fungi in the orders Hypocreales and Entomophthorales are the most commonly used pathogens for biocontrol of insect pests. Biological control strategies using entomopathogenic fungi range from the approach of protecting and enhancing natural enemies already present in the environment to the intentional release of exotic control agents (Eilenberg et al. 2001; Pell et al. 2010). Sustainable control of SWD that do not disrupt currently employed IPM strategies requires the development of new strategies. Undoubtedly, our ecosystems host many pathogenic fungi of unknown value for control of pest populations (Pell et al. 2010), and here we wanted to know if SWD is in the host range of the entomophthoralean fungus E. muscae as a basis for the potential application of entomophthoralean entomopathogens as biocontrol agents. In the present study, we therefore explored the physiological host range by exposing SWD to $E$. muscae from house flies and demonstrated that $E$. muscae is able to infect, behaviourally manipulate and sporulate in SWD.

Higher infectivity and a larger spore size in house flies than in SWD likely illustrates special adaptations of $E$. muscae to the main natural host $M$. domestica. Nevertheless, infected SWD, similar to house flies, showed climbing and posturing of the abdomen with conidiospores growing out between the tergites and sclerites, to get actively discharged. In the natural housefly host, E. muscae disease development is characterized by initial exponential growth (Hansen and De Fine Licht 2017), immune avoidance by proliferating as protoplasts without cell walls (Latge et al. 1988) and behavioural manipulation of hosts to enhance transmission at the final stages of infection (Roy et al. 2006; Gryganskyi et al. 2017). Although less synchronized in time-to-kill, the disease ontogeny and complex behavioural manipulation of $E$. muscae in SWD is similar to infections in housefly. The near- natural E. muscae infection of SWD is consistent with previous work that also documented the potential host range of E. muscae being broader than the known natural host range (Jensen et al. 2006) similar as for E. schizophorae (isolate originally designated E. muscae) that was shown to be infectious for another Drosophila species, D. melanogaster (Steinkraus and Kramer 1987).

Infection of hosts outside the recorded natural host range is known for other infectious pathogens and likely reflects optimized laboratory conditions for pathogen transmission rarely experienced in nature. Under natural conditions, examples of non-host infections are often pathogen spillover events without prolonged ecological persistence in the new host population (Poulin et al. 2011). Remarkably, specific E. muscae isolates have been described to cause high natural infection levels and epizootics in other dipteran pests like the carrot fly Psila rosae or the onion fly Delia antiqua (Carruthers et al. 1985; Eilenberg and Philipsen 1988). Interestingly, carrot flies caught in the hedgerow showed higher infection levels than flies in the adjacent field, illustrating the potential value of E. muscae for control strategies that build on the enhancement of natural enemies in non-crop reservoirs (Eilenberg and Philipsen 1988; Pell et al. 2010). Hedges and other vegetation adjacent to crops are also known as important refuges for SWD (Baroffio et al. 2014; Diepenbrock and Burrack 2017; Kenis et al. 2016), and thus zones where flies may be concealed from conventional pest control strategies used in the field and particularly could get attacked by pathogens and other natural enemies. Moreover, as SWD uses bushes and woods as overwintering sites (Pelton et al. 2016; Briem et al. 2016), a decrease in flies through the presence of pathogens might delay the build-up of dense populations early in season.

As E. muscae is able to infect and kill SWD and furthermore is known to cause epizootics in other dipteran species, it is relevant to contemplate if members of the $E$. muscae species complex could be used in biological control. Attributes that generally are considered as beneficial for the application of entomophthoralean fungi are a specialized 
host range, the potential to cause epizootics and the existence of persisting resting spores (Hajek and Delalibera 2010). However, many Entomophthorales are difficult to mass-produce and grow in vitro (Hajek et al. 2012), and so far devices for autoinoculation have been developed against dipteran pests including SWD only with hypocrealean entomopathogenic fungi (Maniania et al. 2006; Migiro et al. 2010; Yousef et al. 2017). Therefore, control measures operating by release of infected animals that disseminate the pathogens, or enhancement of entomophthoralean fungi in the environment of agroecosystems (e.g. by providing refuges adjacent to crops) might be the most practical way to circumvent the challenging development of formulations for spray application (Tobin and Hajek 2012; Zúbrik et al. 2016). An advantageous attribute for application in pest control is that members of the E. muscae species complex are dipteran-specific, which in comparison with generalist pathogens implies a smaller range of susceptible non-target species. Intricate molecular interactions underlying hostspecific adaptation of $E$. muscae have led to a more narrow host range as compared to generalist hypocrealean fungi such as $M$. robertsii and B. bassiana (De Fine Licht et al. 2017; Hansen and De Fine Licht 2017).

Feasible biological control with the here tested isolate of E. muscae would require a pathogen host shift from the indigenous host species, Musca domestica, on to the invasive SWD. Should a host shift as generated in the laboratory also occur in the field it would be highly beneficial and potentially provide the basis for further development of biological control measures. Other isolates within the E. muscae species complex are known to naturally infect Drosophilid species (Goldstein 1927; Turian and Wüest 1969). Naturally infected species of the genus Drosophila have rarely been collected, but would provide an ideal starting point for developing new diptera-specific biological control strategies for SWD.

\section{Author contribution statement}

PGB and HHDFL designed the study and wrote the manuscript. REJ, MEN and VV performed the assays. REJ and MEN analysed the conidia exposure and morphology data, VV analysed the survival and mortality data. All authors contributed to the writing of the final version of the manuscript.

Acknowledgements The authors thank Jørgen Eilenberg and three anonymous reviewers for comments on an earlier version of the manuscript. HHDFL was supported by a Villum Foundation Young Investigator Grant (Grant Number 10122). PGB was supported by the Swedish Research Council Formas (Grant 2015-1221) and the Swedish University of Agricultural Sciences via the Platform Plant Protection.

\section{Compliance with ethical standards}

Conflict of interest The authors declare that there is no conflict of interest.

Ethical approval Guidelines on ethical issues as well as international agreements were considered and complied with.

Open Access This article is distributed under the terms of the Creative Commons Attribution 4.0 International License (http://crea tivecommons.org/licenses/by/4.0/), which permits unrestricted use, distribution, and reproduction in any medium, provided you give appropriate credit to the original author(s) and the source, provide a link to the Creative Commons license, and indicate if changes were made.

\section{References}

Asplen MK, Anfora G, Biondi A et al (2015) Invasion biology of spotted wing Drosophila (Drosophila suzukii): a global perspective and future priorities. J Pest Sci 88:469-494

Baroffio CA, Richoz P, Fischer S et al (2014) Monitoring Drosophila suzukii in Switzerland in 2012. J Berry Res 4:47-52

Boomsma JJ, Jensen AB, Meyling NV, Eilenberg J (2014) Evolutionary interaction networks of insect pathogenic fungi. Annu Rev Entomol 59:467-485

Briem F, Eben A, Gross J, Vogt H (2016) An invader supported by a parasite: mistletoe berries as a host for food and reproduction of spotted wing Drosophila in early spring. J Pest Sci 89:749-759

Carruthers RI, Haynes DL, MacLeod DM (1985) Entomophthora muscae (Entomophthorales: Entomophthoracae) mycosis in the onion fly, Delia antiqua (Diptera: Anthomyiidae). J Invertebr Pathol 45:81-93

Cini A, Anfora G, Escudero-Colomar LA, Grassi A, Santosuosso U, Seljak G, Papini A (2014) Tracking the invasion of the alien fruit pest Drosophila suzukii in Europe. J Pest Sci 87:559-566

Comont RF, Purse BV, Phillips W et al (2014) Escape from parasitism by the invasive alien ladybird, Harmonia axyridis. Insect Conserv Divers 7:334-342

Cory JS, Myers JH (2000) Direct and indirect ecological effects of biological control. Trends Ecol Evol 15:137-139

Cossentine J, Robertson M, Xu D (2016) Biological activity of Bacillus thuringiensis in Drosophila suzukii (Diptera: Drosophilidae). J Econ Entomol 109:1071-1078

Cox DR (1972) Regression models and life-tables (with discussion). J R Stat Soc B 34:187-220

Cuthbertson A, Audsley N (2016) Further screening of entomopathogenic fungi and nematodes as control agents for Drosophila suzukii. Insects 7:24. doi:10.3390/insects7020024

De Fine Licht HH, Jensen AB, Eilenberg J (2017) Comparative transcriptomics reveal host-specific nucleotide variation in entomophthoralean fungi. Mol Ecol 26:2092-2110

Diepenbrock LM, Burrack HJ (2017) Variation of within-crop microhabitat use by Drosophila suzukii (Diptera: Drosophilidae) in blackberry. J Appl Entomol 141(1-2):1-7

Eilenberg J, Philipsen H (1988) The occurrence of Entomophthorales on the carrot fly [Psila rosae F.] in the field during two successive seasons. Entomophaga 33:135-144

Eilenberg J, Hajek AE, Lomer CJ (2001) Suggestions for unifying the terminology in biological control. Biocontrol 46:387-400

Ferron P (1981) Pest control by the fungi Beauveria and Metarhizium. In: Burges H (ed) Microbial control of pests and plant diseases 1970-1980. Academic Press, London, pp 465-482 
Flexner JL, Lighthart B, Croft BA (1986) The effects of microbial pesticides on non-target, beneficial arthropods. Agric Ecosyst Environ 16:203-254

Goettel MS, Hajek AE (2000) Evaluation of non-target effects of pathogens used for management of arthropods. In: Wajnberg E, Scott J (eds) Evaluating indirect ecological effects of biological control. CABI Publishing, Wallingford, pp 81-97

Goettel MS, Eilenberg J, Glare T (2005) Entomopathogenic fungi and their role in regulation of insect populations. In: Gilbert L, Iatrou $\mathrm{K}$, Gill S (eds) Comprehensive molecular insect science, vol 6. Elsevier, Boston, pp 361-406

Goldstein B (1927) An Empusa disease of Drosophila. Mycologia 19:97-109

Gryganskyi AP, Mullens BA, Gajdeczka MT, Rehner SA, Vilgalys R, Hajek AE (2017) Hijacked: co-option of host behavior by entomophthoralean fungi. PLoS Pathog 13(5):e1006274. doi:10. 1371/journal.ppat.1006274

Hajek AE, Delalibera I (2010) Fungal pathogens as classical biological control agents against arthropods. Biocontrol 55:147-158

Hajek AE, St. Leger RJ (1994) Interactions between fungal pathogens and insect hosts. Annu Rev Entomol 39:293-322

Hajek AE, Papierok B, Eilenberg J (2012) Methods for study of the entomophthorales. In: Manual of techniques in invertebrate pathology, 2nd edn. Elsevier, Amsterdam, p 441-450

Hamby KA, Becher PG (2016) Current knowledge of interactions between Drosophila suzukii and microbes, and their potential utility for pest management. J Pest Sci 89:621-630

Hamby KA, Bellamy DE, Chiu JC, Lee JC, Walton VM, Wiman NG, York RM, Biondi A (2016) Biotic and abiotic factors impacting development, behavior, phenology, and reproductive biology of Drosophila suzukii. J Pest Sci 89:605-619

Hansen AN, De Fine Licht HH (2017) Logistic growth of the hostspecific obligate insect pathogenic fungus Entomophthora muscae in house flies (Musca domestica). J Appl Entomol 141:583-586. doi:10.1111/jen.12380

Haye T, Girod P, Cuthbertson AGS, Wang XG, Daane KM, Hoelmer KA, Baroffio C, Zhang JP, Desneux N (2016) Current SWD IPM tactics and their practical implementation in fruit crops across different regions around the world. J Pest Sci 89:643-651

Jensen AB, Thomsen L, Eilenberg J (2001) Intraspecific variation and host specificity of Entomophthora muscae sensu stricto isolates revealed by random amplified polymorphic DNA, universal primed PCR, PCR-restriction fragment length polymorphism, and conidial morphology. J Invertebr Pathol 78:251-259

Jensen AB, Thomsen L, Eilenberg J (2006) Value of host range, morphological, and genetic characteristics within the Entomophthora muscae species complex. Mycol Res 110:941-950

Jensen AB, Eilenberg J, López Lastra C (2009) Differential divergences of obligately insect-pathogenic Entomophthora species from fly and aphid hosts. FEMS Microbiol Lett 300:180-187

Kalsbeek V, Mullens BA, Jespersen JB (2001) Field studies of Entomophthora (Zygomycetes: Entomophthorales)—Induced behavioral fever in Musca domestica (Diptera: Muscidae) in Denmark. Biol Control 21:264-273

Keane R (2002) Exotic plant invasions and the enemy release hypothesis. Trends Ecol Evol 17:164-170

Keller S (2007) Systematics, taxonomy and identification. In: Keller S (ed) Arthropod-pathogenic entomophthorales: biology, ecology, identification. COST office, Luxembourg, pp 111-126

Keller S, Kalsbeek V, Eilenberg J (1999) Redescription of Entomophthora muscae (Cohn) Fresenius. Sydowia 51:197-209

Kenis M, Tonina L, Eschen R et al (2016) Non-crop plants used as hosts by Drosophila suzukii in Europe. J Pest Sci 89:735-748

Latge JP, Eilenberg J, Beauvais AI, Prevost MC (1988) Morphology of Entomophthora muscae protoplasts grown in vitro. Protoplasma 146:166-173
Maniania NK, Ekesi S, Odulaja A et al (2006) Prospects of a funguscontamination device for the control of tsetse fly Glossina fuscipes fuscipes. Biocontrol Sci Technol 16:129-139

Meyling NV, Eilenberg J (2007) Ecology of the entomopathogenic fungi Beauveria bassiana and Metarhizium anisopliae in temperate agroecosystems: potential for conservation biological control. Biol Control 43:145-155

Migiro LN, Maniania NK, Chabi-Olaye A, Vandenberg J (2010) Pathogenicity of entomopathogenic fungi Metarhizium anisopliae and Beauveria bassiana (Hypocreales: clavicipitaceae) isolates to the adult pea leafminer (Diptera: Agromyzidae) and prospects of an autoinoculation device for infection in the field. Environ Entomol 39:468-475

Pell JK, Hannam JJ, Steinkraus DC (2010) Conservation biological control using fungal entomopathogens. Biocontrol 55:187-198

Pelton E, Gratton C, Isaacs R et al (2016) Earlier activity of Drosophila suzukii in high woodland landscapes but relative abundance is unaffected. J Pest Sci 89:725-733

Poulin R, Krasnov BR, Mouillot D (2011) Host specificity in phylogenetic and geographic space. Trends Parasitol 27:355-361

R Core Team (2013) R: a language and environment for statistical computing. $\mathrm{R}$ foundation for statistical computing, Vienna. http://www.R-project.org/

Revadi S, Lebreton S, Witzgall P et al (2015) Sexual behavior of Drosophila suzukii. Insects 6:183-196

Roberts DW, Humber RA (1981) Entomogenous fungi. In: Cole GT, Kendrick B (eds) Biology of conidial fungi, vol 2. Academic Press, New York, pp 201-236

Roy HE, Steinkraus DC, Eilenberg J et al (2006) Bizarre interactions and endgames: entomopathogenic fungi and their arthropod hosts. Annu Rev Entomol 51:331-357

Shah PA, Pell JK (2003) Entomopathogenic fungi as biological control agents. Appl Microbiol Biotechnol 61:413-423

Steinkraus DC, Kramer JP (1987) Susceptibility of sixteen species of Diptera to the fungal pathogen Entomophthora muscae ( $\mathrm{Zy}-$ gomycetes: Entomophthoraceae). Mycopathologia 100:55-63

Tobin PC, Hajek AE (2012) Release, establishment, and initial spread of the fungal pathogen Entomophaga maimaiga in island populations of Lymantria dispar. Biol Control 63:31-39

Turian G, Wüest J (1969) Mycoses a Entomophthoracees frappant des populations de fourmis et de Drosophiles. Mitt Schweiz Entomol Ges 42:197-201

Vega FE, Meyling NV, Luangsa-Ard JJ, Blackwell M (2012) Fungal entomopathogens, 2nd edn. Elsevier, Amsterdam

Vestergaard S, Cherry A, Keller S, Goettel MS (2003) Safety of Hyphomycete fungi as microbial control agents. In: Hokkanen HMT, Hajek A (eds) Environmental impacts of microbial insecticides: need and methods for risk. Kluwer Academic, Boston, pp 35-62

Wang C, Wang S (2017) Insect pathogenic fungi: genomics, molecular interactions, and genetic improvements. Annu Rev Entomol 62:73-90

Woltz JM, Donahue KM, Bruck DJ, Lee JC (2015) Efficacy of commercially available predators, nematodes and fungal entomopathogens for augmentative control of Drosophila suzukii. J Appl Entomol 139:759-770

Yousef M, Aranda-Valera E, Quesada-Moraga E (2017) Lure-andinfect and lure-and-kill devices based on Metarhizium brunneum for spotted wing Drosophila control. J Pest Sci. doi:10.1007/ s10340-017-0874-8

Zúbrik M, Hajek A, Pilarska D et al (2016) The potential for Entomophaga maimaiga to regulate gypsy moth Lymantria dispar (L.) (Lepidoptera: Erebidae) in Europe. J Appl Entomol 140:565-579 